\title{
Novel Simulation Algorithms for Developing Measuring Techniques for Multichannel Ultrasonic Microscopy
}

\author{
Mario Wolf', Elfgard Kühnicke ${ }^{1}$ \\ 1 Solid State Electronics Laboratories, TU Dresden, Mommsenstraße 1501069 Dresen, Germany \\ Corresponding author's Mario.wolf@tu-dresden.de
}

\begin{abstract}
:
New ultrasonic measuring techniques use information from local distribution of reflected waves gained with arrays. As they are taking the wave behavior of sound into account, simple geometric models are not sufficient to understand the dependence between quantities being measured directly and these quantities being of interest, like sound velocity in media or the geometry of a specimen. To get this understanding, sound field simulations are necessary which allow to derive evaluation algorithm and to qualify measuring results. To reduce the calculation-effort for axial symmetric problems this contribution presents a new approach to calculate the sound field of a ring-like source. The results are proved by comparing them with the sound field of a disc shaped source.
\end{abstract}

Key words: ultrasound, sound field calculation, Green's functions, ring-like excitation, annular array

\section{Introduction}

New ultrasonic measurement techniques utilize the wave behavior of sound instead evaluating only amplitude and/or time of flight. So e.g. new algorithm for synthetic aperture focusing technique take into account the sound field of the transducer instead of simple delay and sum [1]. In [2] the authors developed new, nontomographic methods for a simultaneous measurement of sound velocity and thickness of layered structures and for a locally resolved measurement of sound velocity in fluids with scattering particles. These techniques analyze the local distribution of reflected waves to gain additional information. This is done with structured transducers, in [2] with annular arrays, consisting of a circular disc as central element and rings of the same area, see figure 1 .

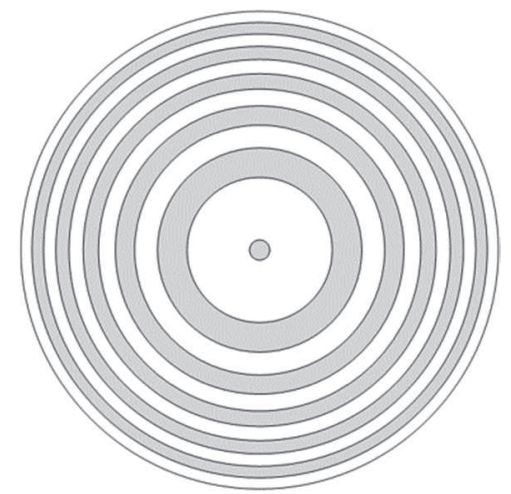

Fig. 1. Example of structure of the used annular arrays: white - active area, grey passive area.
As the new measuring techniques are used to analyze very complex problems, a prediction of reflected signals, via a simulation, is necessary to gain evaluation methods and to qualify measurement results. Half-analytic methods are a powerful tool to model wave propagation in complex structures. They are based on integral transform methods, which are used to derive Green's functions giving the pulse response of a point-like excitation on the interface between two half-spaces. So in comparison to full-numerical methods, based on finite elements, not the whole volume but only the interfaces need to be discretized. So the effort of memory and computation time becomes much lower. Especially for the simulation of ultrasonic problems a very high discretization is required but there are often even big volumes which has to be taken into account.

Integral transform methods are used to reduce multidimensional differential equations to ordinary ones, as it is known from the mostly widespread Fourier Transform. The transformed equation can be solved simply by an exponential ansatz and the integration constants are determined by applying the boundary conditions. So a correct analytic solution can be derived in the transformed domain. The main issue is performing the inverse transform. This can be done by the Cangiard-de Hoop method as in [3] gaining the step response in time domain or by a steepest descent approximation gaining the field 
of a point source with directivity pattern in frequency domain [4].

As annular arrays are used in the developed measuring techniques, most simulated problems are radial-symmetric. Thus modelling can be done in two instead of three dimensions, which would reduce the computation effort significantly. This contribution describes the derivation of Greens functions for ring-like sources. It discusses why applying the steepest descent approximation fails and how an alternative approach using Green's functions of point sources in the frequency domain can be used to calculate the pulse response of a ringlike source.

\section{Derivation of Green's Functions}

Axial-symmetric problems are described by the wave equation in cylinder coordinates (eq. 1) for the potential $\Phi$ of displacement:

$$
\frac{\partial^{2}}{\partial r^{2}} \Phi+\frac{1}{r} \frac{\partial}{\partial r} \Phi+\frac{\partial^{2}}{\partial z^{2}} \Phi-\frac{1}{c_{L}^{2}} \frac{\partial^{2}}{\partial t^{2}} \Phi=0
$$

To solve this equation, the Fourier transform is applied with respect to time and the Hankel transform with respect to the radial coordinate $r$.

$$
H_{0}(\xi)=H(h(r))=\int_{0}^{\infty} h(r) J_{0}(r \xi) r d r
$$

where $J_{0}$ is the Bessel function of first kind and zeroth order. So an ordinary differential equation

$$
\frac{\partial^{2}}{\partial z^{2}} \Phi^{H_{0}}+\left(\xi^{2}+k^{2}\right) \Phi^{H_{0}}=0
$$

is gained, with the wave number $k$. This equation can be solved by an exponential ansatz.

$\Phi^{H_{0}}=A e^{-j \sqrt{\xi^{2}+k^{2}} z}+B e^{j \sqrt{\xi^{2}+k^{2}} z}$

To determine $A$ and $B$ the boundary conditions has to be taken into account. The normal stress for a point source is given by:

$$
\sigma_{z z}(r)=\sigma_{0} \frac{\delta(r)}{2 \pi r}
$$

and

$\sigma_{z z}^{H_{0}}(\xi)=\sigma_{0}$

in the transformed domain. For a ring-like excitation, at the position $r=a$, it is given by

$\sigma_{z z}(r)=\sigma_{0} \frac{\delta(r-a)}{2 \pi(r-a)}$

and can also be transformed easily:

$$
\sigma_{z z}^{H_{0}}(\xi)=\sigma_{0} J_{0}(a \xi)
$$

Additionally, stress and displacement at the interface must be equal. For a point source, independently if stress or displacement components are calculated, an integral of the form:

$$
I=\int_{0}^{\infty} F(\xi) e^{-j \sqrt{k^{2}+\xi^{2}} z} J_{0}(r \xi) \xi d \xi
$$

has to be solved for the inverse transform. For the ring-like excitation the integral looks very similar, only an additional Bessel function appears.

$$
I=\int_{0}^{\infty} F(\xi) e^{-j \sqrt{k^{2}+\xi^{2}} x} J_{0}(a \xi) J_{0}(r \xi) \xi d \xi
$$

Trying to solve this by substituting $F^{*}=F J_{0}(a \xi)$ would fail immediately because this would result in a function with an epicenter at the coordinate origin whereas it has to be at $r=a$. To obtain this, first the integral form of a Bessel function is required:

$$
\begin{aligned}
J_{0(r \xi)} & =\frac{1}{2 \pi} \int_{0}^{\infty} \frac{1}{\sqrt{j x^{2}+2 r \xi}} e^{-x^{2}+j r \xi} d x \\
& +\frac{1}{2 \pi} \int_{0}^{\infty} \frac{1}{\sqrt{j x^{2}-2 r \xi}} e^{-x^{2}-j r \xi} d x
\end{aligned}
$$


The multiplication of the two Bessel functions results in a sum of four terms which can be combined to a sum of two terms. Approximating each term as in [4] with a linear approximation results in the integral of eq. (12):

$$
\begin{aligned}
I= & \frac{1}{\pi} \int_{-\infty}^{\infty} \frac{F(\xi)}{2 \xi \sqrt{a r}} e^{-p z+j(r+a) \xi+\frac{\pi}{2}} \xi d \xi \\
& +\frac{1}{\pi} \int_{-\infty}^{\infty} \frac{F(\xi)}{2 \xi \sqrt{a r}} e^{-p z+j(r-a) \xi+\frac{\pi}{2}} \xi d \xi
\end{aligned}
$$

Note that this equation has the epicenter at $r=a$, and $r=-a$. Applying the steepest descent approximation on these integrals gives:

$$
\begin{aligned}
& I_{1}=\frac{F\left(\xi_{s}\right) \cos (\alpha)}{\sqrt{2 \pi k R a(R \sin (\alpha)-a)}} e^{-j k R} \\
& +\frac{F\left(\xi_{s}^{\prime}\right) \cos \beta}{\sqrt{2 \pi k R^{\prime} a\left(R^{\prime} \sin (\beta)+a\right)}} e^{-j k R^{\prime}}
\end{aligned}
$$

, with

$$
\begin{array}{ll}
R=\sqrt{(r-a)^{2}+z^{2}} & \\
R^{\prime}=\sqrt{(r+a)^{2}+z^{2}} & \\
\alpha=\arctan \left(\frac{r-a}{z}\right) & \beta=\arctan \left(\frac{r+a}{z}\right) \\
\xi_{s}=k \sin (\alpha) & \xi_{s}^{\prime} k \sin (\beta)
\end{array}
$$

These formulas are implemented and the resulting field is compared with the corresponding one, gained by a point source synthesis. The results are shown in figure 2 .

Obviously both fields disagree. The reason is, that the two Bessel functions had been approximated linearly and that the resulting deviation becomes too high. So an alternative approach is proposed:
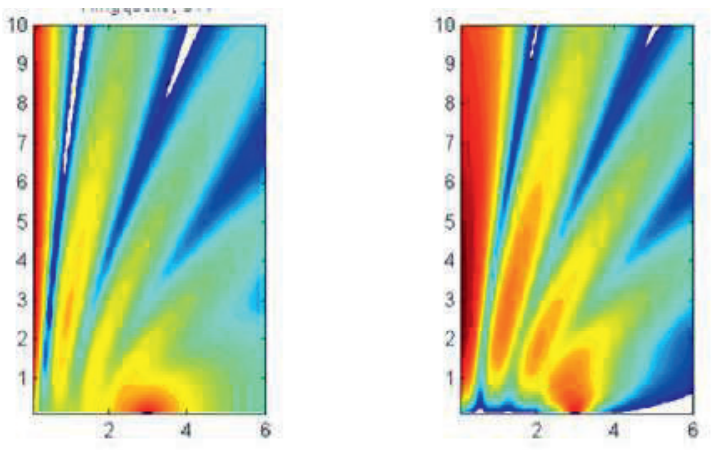

Fig. 2. Comparison of calculated sound fields of a point source at $r=3 \mathrm{~mm}$ : via the steepest descent approximation for the ring (left) and via point source synthesis (right).

\section{Approach using transient Green's functions of point sources}

The field of a point source gained from the steepest descent approximation is given by

$G=\frac{S(\varphi)}{2 \pi R} e^{j k R}$

, where $R$ is the distance between source and observation point, $S$ is the directivity pattern, determined by the boundary conditions and the excitation type, $\varphi$ is the angle between $R$ and the surface normal at the source point. For a source point at $P_{S}(a \cos \alpha, a \sin \alpha, 0)$ and an observation point at $P_{0}(x, 0, z)$ the pressure is given in eq. (16) and illustrated in figure 3.

$$
\begin{aligned}
p(x, z)= & \frac{p_{0} S(\varphi)}{2 \pi \sqrt{\left(x^{2}-2 x a \cos (\alpha)+a^{2}+z^{2}\right)}} \\
& \cdot e^{j k \sqrt{x^{2}-2 x a \cos (\alpha)+a^{2}+z^{2}}}
\end{aligned}
$$

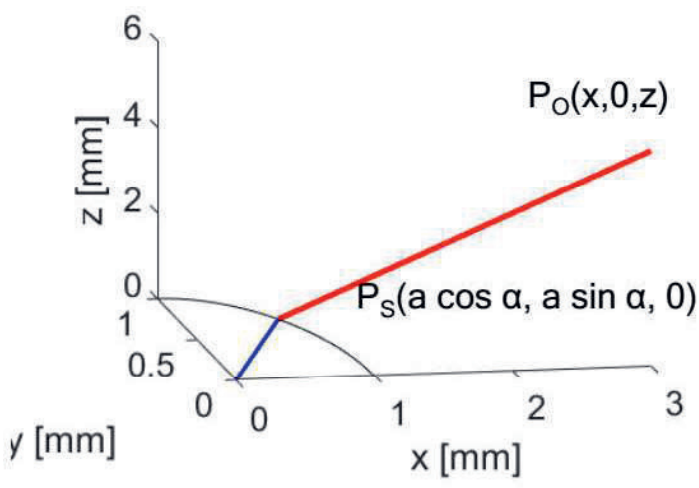

Fig. 3. View of the source and an arbitrary observation point. 
This can be transformed back into time domain, where we get a Dirac function instead of the exponential term. To calculate the field of a ringlike source we have to integrate over $\alpha$, as in eq. (17)

$$
\begin{aligned}
& p_{\text {Ring }}=\int_{0}^{\pi} \frac{a p_{0} S(\varphi)}{\pi \sqrt{x^{2}+a^{2}+z^{2}-2 x a \cos (\alpha)}} \\
& \cdot \delta\left(t-\frac{\sqrt{x^{2}+a^{2}+z^{2}-2 x a \cos (\alpha)}}{c}\right) d \alpha
\end{aligned}
$$

Due to the properties of $\delta$-distribution, this integral can be solved: We substitute

$t^{\prime}=\frac{\sqrt{\left(x^{2}-2 x a \cos (\alpha)+a^{2}+z^{2}\right)}}{c}$

And obtain, with the step function $H(t)$.

$$
\begin{aligned}
& p(x, z)=\frac{2 a p_{0} S(\varphi)\left(H\left(t-t_{1}\right)-H\left(t-t_{2}\right)\right)}{\pi \sqrt{4 a^{2} x^{2}-\left(x^{2}+a^{2}+z^{2}-c^{2} t^{2}\right)^{2}}} \\
& \text { with } \varphi=\arccos \left(\frac{z}{c t}\right) \quad \text { (19) }
\end{aligned}
$$

for $x>0$ and the integration constants:

$$
\begin{aligned}
& t_{1}=\frac{\sqrt{\left(x^{2}-2 x a+a^{2}+z^{2}\right)}}{c} \\
& t_{2}=\frac{\sqrt{\left(x^{2}+2 x a+a^{2}+z^{2}\right)}}{c}
\end{aligned}
$$

For the pressure on the axis we obtain.

$$
p(0, z)=\frac{p_{0} S(\varphi) \delta\left(t-\frac{\sqrt{a^{2}+z^{2}}}{c}\right)}{\pi \sqrt{a^{2}+z^{2}}}
$$

So the special pulse response for a ring-like excitation on an interface of two half-spaces can be calculated in the whole volume.

\section{Results and Discussions}

To prove the derived equations, they had been used to calculate the sound field of a disc shaped source as in [5]. Thereby it has to be recognized that the approach of the steepest descent approximation separates the pressure and sheer waves and that they have to be calculated independently. But in [5] the pulse response is calculated by the Cangiard-de Hoop method what results in an term containing both wave types. It calculates directly e.g. the normal displacement.

The calculated signals for a disc-source of $10 \mathrm{~mm}$ diameter are depicted in figure 4 (blue lines). For the pressure on the acoustic axis the expected rectangular signal can be observed. If the observation point is not on the axis but its radial coordinate is lower than the radius of the disc, a much shorter rectangular signal part with a decaying edge can be observed. The time, when the decay starts is the arrival-time of the first edge wave. If the radial coordinate of the observation point is bigger than the disc diameter, there is no rectangular signal part. The Figure also shows the signals which would be emitted of a transducer with a center frequency of $3 \mathrm{MHz}$ if it is excited with a signal of 3 Periods (red lines).
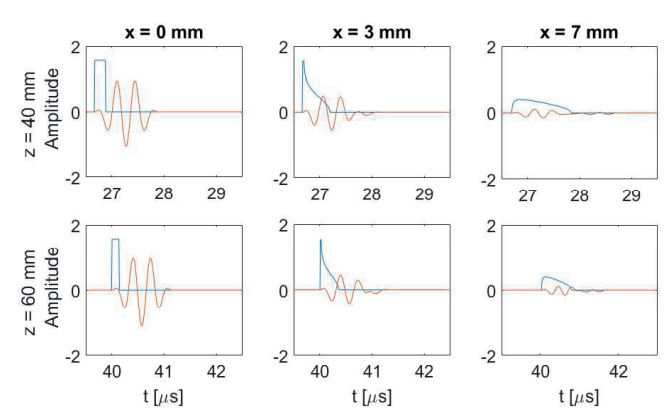

Fig. 4. Calculated signals for a disc source of $d=10 \mathrm{~mm}$ diameter for different depths $(40 \mathrm{~mm}$ upper row and $60 \mathrm{~mm}$ lower row) and for different radial coordinates $r$; left: on acoustic axis, center: out of axis $r<d / 2$, right: $r>d / 2$; blue: pulse response; red convolution with a signal of 3 periods with $3 \mathrm{MHz}$ center frequency.

To determine the necessary density of rings, calculations are done for different densities. Some examples are shown in figure 5 . It can be recognized that the distance of two rings may not be larger than $1 \mu \mathrm{m}$. 


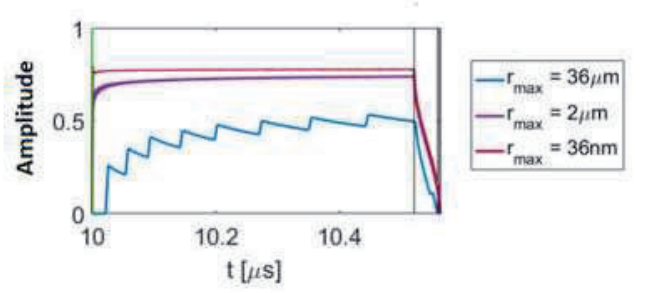

Fig. 5 Calculated signals for a disc source of $d=10 \mathrm{~mm}$ diameter for different ring densities.

The proposed method can be used to calculate the sound fields of annular arrays very effectively. Therefore, pulse responses are calculated in each observation point and are convoluted with an arbitrary excitation pulse. For visualization, the maximal amplitude of the resulting signals is shown in figure 6 for an array of 6 elements with a diameter of the outer ring of $d=12 \mathrm{~mm}$. All elements have the same area and there is no space between them. The elements are represented by bold lines on the $\mathrm{x}$-axis.

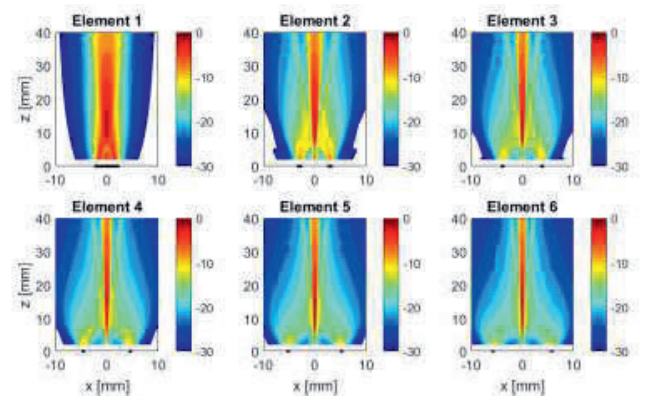

Fig.6 Calculated maximal amplitude of the single elements of a 6-element-annular array, in $d B$ standardized to its own maximum.

The big advantage of annular arrays are their excellent properties for focusing, as the resulting focus has a small extension in all directions. It can be placed between the near field length of a single element and the near field length of the whole transducer, driving all elements together. To focus electronically in measurements, the elements are driven quasi-simultaneously, which means that there is a small delay between the signals but this delay is usually smaller than the length of the signal. The delay times are calculated by determining the different path lengths from each element to the assigned focus position and dividing these differences by the sound velocity of propagation medium. So each wave arrives at the assigned point at the same time. This can also be realized very easily in simulations as the calculated signals can be time-shifted and superposed. Some examples are shown in figure 7. The upper row is for excitation with $2 \mathrm{MHz}$ and the lower row for excitation with $4 \mathrm{MHz}$, always with a signal of 3 periods. On the left there is no focusing so that the resulting field has a focus outside the observation area. In the center the fields are shown for trying to focus at a distance of $10 \mathrm{~mm}$ what succeeds for $2 \mathrm{MHz}$ but fails for $4 \mathrm{MHz}$ because the nearfield length of the single element is there already at $15 \mathrm{~mm}$ which makes focusing impossible

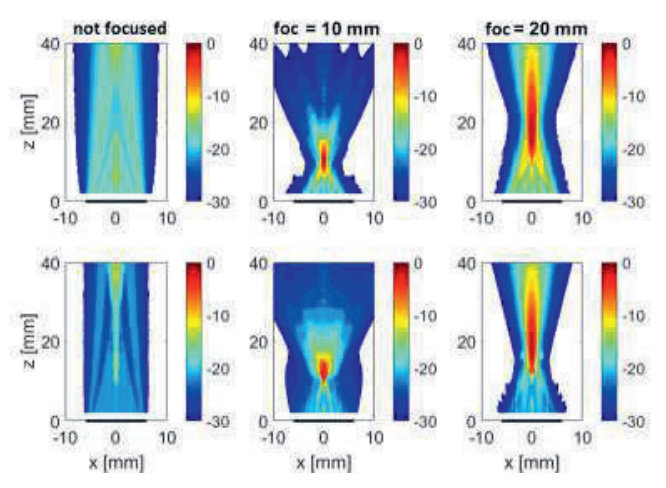

Fig.7 Calculated sound fields resulting from focusing for the array of figure 6 but with excitation of $2 \mathrm{MHz}$ (top) and $4 \mathrm{MHz}$ (bottom) for: no focusing (left) focusing on $10 \mathrm{~mm}$ (center) and $20 \mathrm{~mm}$ (right), in $\mathrm{dB}$ standardized to its own maximum.

\section{Summary and Conclusion}

This contribution gives a new approach to simulate the sound propagation in radialsymmetric structures. Therefor the pulse response for a ring-like excitation has been derived. This allows to discretize the interfaces of the structures only in one dimension instead of two, which had been necessary for the point source synthesis. Although it neglects the waves which are emitted from surface waves it predicts the signals of transducers very well.

Future work will be on the optimization of the discretization of the interface for best accuracy at a minimal calculation time. Furthermore, the algorithm will be extended for problems of several layers.

\section{Acknowledgment}

The authors would like to thanks the Deutsche Forschungsgemeinschaft (DFG) for financial support in the project: KU 1075/18-1

\section{References}

[1] Spies, Martin, et al. "Synthetic aperture focusing and time-of-flight diffraction ultrasonic imagingpast and present." Journal of Nondestructive Evaluation 31.4 (2012): 310-323.

[2] Wolf, Mario, et al. "Annular arrays for novel ultrasonic measurement techniques." Journal of Sensors and Sensor Systems 5.2 (2016): 373.

[3] Pao, Yih-Hsing, and R. R. Gajewski. "The generalized ray theory and transient responses of 
layered elastic solids." Physical acoustics 13 (1977): 183-265.

[4] Miller, G. F., and H. Pursey. "The field and radiation impedance of mechanical radiators on the free surface of a semi-infinite isotropic solid." Proceedings of the Royal Society of London A. Mathematical, Physical and Engineering Sciences. Vol. 223. No. 1155. The Royal Society, 1954.

[5] Bresse, L.F. and Hutchins, D. A.: Transient generation of elastic waves in solids by a diskshaped normal force source. The Journal of the Acoustical Society of America 86-2 (1989), 810817 